\title{
The minimum record time for PIV measurement in a vessel agitated by a Rushton turbine
}

\author{
Radek Šulc ${ }^{1, *}$, Pavel Ditl ${ }^{1}$, Ivan Fořt ${ }^{1}$, Darina Jašíkova ${ }^{2}$, Michal Kotek $^{2}$, Václav Kopecký ${ }^{2}$, and Bohuš Kysela ${ }^{3}$ \\ ${ }^{1}$ Czech Technical University in Prague, Faculty of Mechanical Engineering, Department of Process Engineering, Technická 4, 16607 \\ Prague, Czech Republic \\ ${ }^{2}$ Technical University of Liberec, Institute for Nanomaterials, Advanced Technology and Innovation, Studentská 1402/2, 461 17 Liberec, \\ Czech Republic \\ ${ }^{3}$ Czech Academy of Sciences, Institute of Hydrodynamics v.v.i., Pod Pat'ankou 30/5, 16612 Prague, Czech Republic
}

\begin{abstract}
In PIV studies published in the literature focusing on the investigation of the flow field in an agitated vessel the record time is ranging from the tenths and the units of seconds. The aim of this work was to determine minimum record time for PIV measurement in a vessel agitated by a Rushton turbine that is necessary to obtain relevant results of velocity field. The velocity fields were measured in a fully baffled cylindrical flat bottom vessel $400 \mathrm{~mm}$ in inner diameter agitated by a Rushton turbine $133 \mathrm{~mm}$ in diameter using 2-D Time Resolved Particle Image Velocimetry in the impeller Reynolds number range from 50000 to 189000 . This Re range secures the fully-developed turbulent flow of agitated liquid. Three liquids of different viscosities were used as the agitated liquid. On the basis of the analysis of the radial and axial components of the mean- and fluctuation velocities measured outside the impeller region it was found that dimensionless minimum record time is independent of impeller Reynolds number and is equalled N.t $\mathrm{R}_{\mathrm{Rin}}=$ $103 \pm 19$.
\end{abstract}

\section{Introduction}

The knowledge of the flow and the flow pattern in an agitated vessel is important for determination of many impeller and turbulence characteristics, e.g. impeller pumping capacity, intensity of turbulence, turbulent kinetic energy, convective velocity, and the turbulent energy dissipation rate. The information and data that are obtained can also be used for CFD verification.

The hydrodynamics and flow field are investigated in an agitated vessel usually using Particle Image Velocimetry (PIV) or Laser-Dopler Anemometry (LDA). In PIV studies published in the literature the record time is ranging from the tenths and the units of seconds. Kysela et al. [1] investigated the hydrodynamics in a fully baffled cylindrical flat bottom vessel $300 \mathrm{~mm}$ in inner diameter filled by water agitated by a Rushton turbine with impeller speed $300 \mathrm{rpm}$. They analysed time record of radial mean- and fluctuation velocities measured in the impeller discharge flow. They reported that the analysed velocities were relatively stable values after $5 \mathrm{~s}$ of the record. From this study, it follows that the record time is one of the important factors affecting the relevance and accuracy of measured data. However, it should be noted that this result was observed only for one liquid and one impeller speed.

The aim of this work is to determine minimum record time for PIV measurement in an agitated vessel that is necessary to obtain relevant results of velocity field. The hydrodynamics and flow field were measured in an agitated vessel using 2-D Time Resolved Particle Image Velocimetry (2-D TR PIV). Three liquids of different viscosities were used as the agitated liquid: i) distilled water $\left(v=9.35 \times 10^{-7} \mathrm{~m}^{2} / \mathrm{s}\right)$, ii) a $28 \%$ vol. aqueous solution of glycol $\left(v=2 \times 10^{-6} \mathrm{~m}^{2} / \mathrm{s}\right)$, and iii) a $43 \%$ vol. aqueous solution of glycol $\left(v=3 \times 10^{-6} \mathrm{~m}^{2} / \mathrm{s}\right)$. The velocity fields were measured at an impeller rotation speed in the range from $300 \mathrm{rpm}$ to $850 \mathrm{rpm}$, which covers the impeller Reynolds number range from 50000 to 189 000. This Re range secures the fully-developed turbulent flow of agitated liquid.

\section{Theoretical background}

For highly turbulent flow of Newtonian fluid in a baffled vessel, the viscous and gravitational forces can be neglected and finally the time-averaged dimensionless velocity components are independent of the Reynolds number and the Froude number, and depend on the location only.

Using PIV, the instantaneous velocity data set $U_{i}\left(t_{j}\right)$ in the $\mathrm{i}^{\text {th }}$ direction for $j=1,2, \ldots N_{R}$ at observation times $t_{j}$ with equidistant time step $\Delta t_{E}$ (i.e. $\Delta t_{E}=t_{j+1}-t_{j}$ ) was obtained in a given location. Using obtained data set $U_{i}$ $\left(t_{j}\right)$ in the $\mathrm{i}^{\text {th }}$ direction the time-averaged mean velocity $\bar{U}_{i}$ and root mean squared fluctuation velocity $\bar{u}_{i}$ were calculated in given direction for averaging interval of various lengths $t_{P}$. The time-averaged mean velocity

\footnotetext{
Corresponding author: radek.sulc@fs.cvut.cz
} 
$\bar{U}_{i}\left(t_{p}\right)$ for averaging interval of time length $t_{P}$ was determined using the ergodic hypothesis as the average value of velocity data items $U_{i}\left(t_{j}\right)$ in the averaging interval of time length $t_{P}$ :

$$
\bar{U}_{i}\left(t_{P}\right)=\frac{1}{N_{P}} \cdot \sum_{j} U\left(t_{j}\right) \text { for } j=1,2, \ldots, N_{P}
$$

where $\bar{U}_{i}\left(t_{P}\right)$ is mean velocity in the $\mathrm{i}^{\text {th }}$ direction for averaging interval $t_{P}, U_{i}\left(t_{j}\right)$ is instantaneous velocity in the $i^{\text {th }}$ direction at the observation time $t_{j}$, and $N_{P}$ is the number of data items in velocity data set for averaging interval of time length $t_{P}$.

Consequently, the fluctuation velocity in the $i^{\text {th }}$ direction $u_{i}\left(t_{j}\right)$ at observation time $t_{j}$ for averaging interval of time length $t_{P}$ is obtained by decomposition of the instantaneous velocity:

$$
u_{i}\left(t_{j}\right)=U_{i}\left(t_{j}\right)-\bar{U}_{i}\left(t_{P}\right) \text { for } j=1,2, \ldots, N_{P}
$$

where $u_{i}\left(t_{j}\right)$ is the fluctuation velocity in the $\mathrm{i}^{\text {th }}$ direction at observation time $t_{i}, \bar{U}_{i}\left(t_{P}\right)$ is mean velocity in the $i^{\text {th }}$ direction for averaging interval $t_{P}$ and $U_{i}\left(t_{i}\right)$ is instantaneous velocity in the $i^{\text {th }}$ direction at the observation time $t_{i}$.

The root mean squared fluctuation velocity for averaging interval of time length $t_{P}$ is determined as follows:

$$
\bar{u}_{i}\left(t_{P}\right)=\left(\frac{1}{N_{P}} \cdot \sum_{i} u_{i}^{2}\left(t_{j}\right)\right)^{1 / 2} \text { for } j=1,2, \ldots, N_{P},
$$

where $\bar{u}_{i}\left(t_{P}\right)$ is the root mean squared fluctuation velocity in the $i^{\text {th }}$ direction for averaging interval $t_{P}$, and $u\left(t_{i}\right)$ is the fluctuation velocity at observation time $t_{i}$ for time interval $t_{P}$.

\section{Experimental}

The hydrodynamics and the flow field were measured in an agitated vessel using Time Resolved Particle Image Velocimetry (TR PIV). The experiments were carried out in a fully baffled cylindrical flat bottom vessel 400 $\mathrm{mm}$ in the inner diameter. The tank was provided with a Rushton turbine $133 \mathrm{~mm}$ in diameter, i.e. the dimensionless impeller diameter $D / T$ was $1 / 3$. The dimensionless impeller clearance $C / D$ taken from the lower impeller edge was 0.75 . The liquid height was 400 $\mathrm{mm}$, i.e. the dimensionless liquid height $H / T$ was 1 . The dimensionless baffle width $B / T$ was $1 / 10$. To prevent air suction, the vessel was covered by a lid.

Three liquids of different viscosities were used as the agitated liquid: i) distilled water $\left(v=9.35 \times 10^{-7} \mathrm{~m}^{2} / \mathrm{s}\right)$, ii) a $28 \%$ vol. aqueous solution of glycol $\left(v=2 \times 10^{-6} \mathrm{~m}^{2} / \mathrm{s}\right)$, and iii) a $43 \%$ vol. aqueous solution of glycol $\left(v=3 \times 10^{-}\right.$ ${ }^{6} \mathrm{~m}^{2} / \mathrm{s}$ ). The velocity fields were measured at an impeller rotation speed in the range from $300 \mathrm{rpm}$ to $850 \mathrm{rpm}$, which covers the impeller Reynolds number range from 50000 to 189000 . This means that fully-developed turbulent flow of agitated liquid was reached. The operational conditions are summarized in Table 1.

The time-resolved LITRON LDY 302 2D-PIV system (Dantec Dynamics (Denmark)) consists of a Neodyme-YLF laser (light wave length $532 \mathrm{~nm}$, impulse energy 2x15 mJ), a SpeedSence 611 high speed PIVregime camera (working on frequency $1 \mathrm{kHz}$ with resolution 1280 x 800 pixels) with a Nikon Macro 200 optical lens system equipped with an optical filter of wave length $570 \mathrm{~nm}$. Rhodamine B fluorescent particles $11.95 \pm 0.25 \mu \mathrm{m}$ in the mean diameter were used as seeding particles. The fluorescent particles lit by $532 \mathrm{~nm}$ light emit $570 \mathrm{~nm}$ light. In this way, non-seeding particles such as impurities and bubbles are separated, and are not recorded. The operating frame rate was 1 $\mathrm{kHz}$ (1000 vector fields per second), i.e. the sampling time $\Delta \mathrm{t}_{\mathrm{s}}$ was $1 \mathrm{~ms}$. The measured vertical plane was located in the center of the vessel and in the middle of the baffles. The plane was illuminated by a laser sheet. The investigated area was $43 \times 27 \mathrm{~mm}$, and the PIV spatial resolution $\Delta=0.74 \mathrm{~mm}$ was maintained the same during the experiment.

The scheme of the experimental apparatus and the investigated area is depicted in Fig. 1. The camera was positioned orthogonally to the laser sheet. The investigated area corresponds to region $\mathrm{F}$ according to the classification given by Fořt et al. [2]. The method of an ensemble averaged velocity field can be used due to axisymmetric character of the flow in this region. The selected data obtained in position $\mathrm{P}$ corresponding to dimensionless radius $2 r / T=0.224$ and dimensionless height $z / T=0.138$ are presented in this paper.
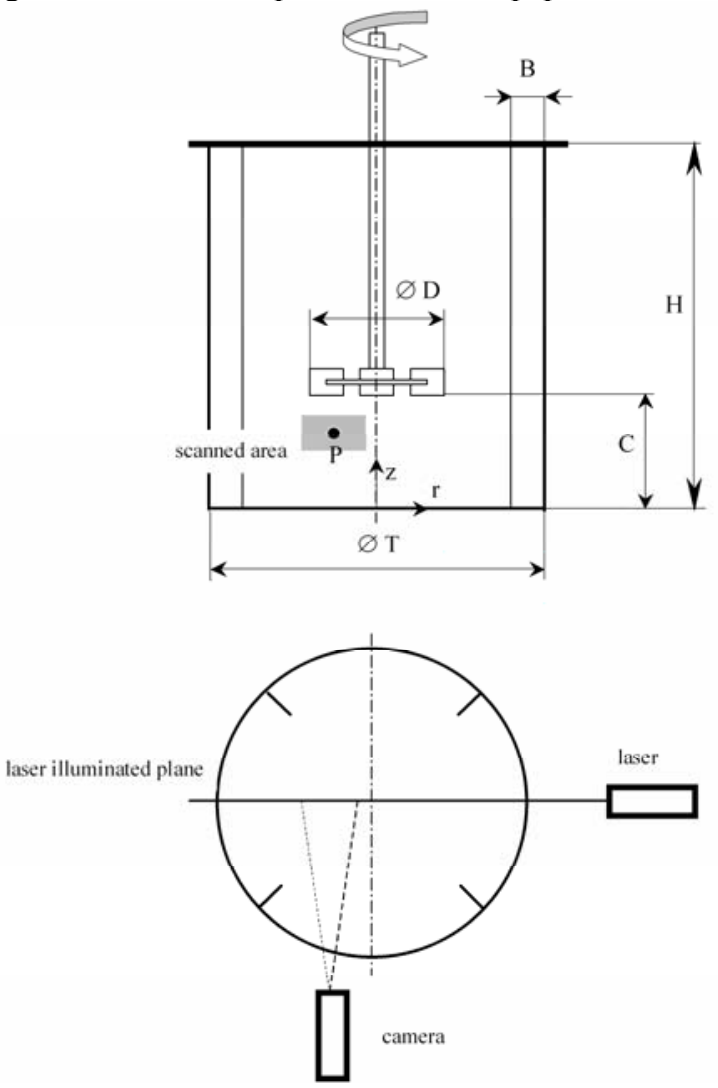

Fig. 1. Scheme of the experimental apparatus and the investigated area. 
Table 1. Operational parameters.

\begin{tabular}{|c|c|c|c|c|c|}
\hline Run \# & liquid & $\begin{array}{c}\mathrm{N} \\
(\mathrm{rpm})\end{array}$ & $\begin{array}{c}\mathrm{v} \\
\left(\mathrm{m}^{2} / \mathrm{s}\right)\end{array}$ & $\begin{array}{c}\text { Re } \\
(-)\end{array}$ & $\begin{array}{c}\text { Record time } \mathrm{T}_{\mathrm{R}} \\
(\mathrm{s})\end{array}$ \\
\hline w-N300 & water & 300 & $9.35 \times 10^{-7}$ & 94641 & 24.8 \\
W-N600 & water & 600 & $9.35 \times 10^{-7}$ & 189282 & 24.8 \\
g28-N600 & $28 \%$ glycol & 600 & $2 \times 10^{-6}$ & 88445 & 24.8 \\
g28-N750 & $28 \%$ glycol & 750 & $2 \times 10^{-6}$ & 110556 & 24.8 \\
g43-N600 & $43 \%$ glycol & 600 & $3 \times 10^{-6}$ & 58963 & 24.8 \\
g43-N750 & $43 \%$ glycol & 750 & $3 \times 10^{-6}$ & 73704 & 24.8 \\
g43-N850 & $43 \%$ glycol & 850 & $3 \times 10^{-6}$ & 83531 & 24.8 \\
\hline
\end{tabular}

\section{Experimental data evaluation}

The time courses of mean- and fluctuation velocities in radial and axial direction calculated for averaging intervals of various time lengths are presented in Figures 2 - 8 for each experimental run. The time courses of radial mean velocity are close to zero and have irregular shape. These findings correspond to characteristics of the given zone, according to Fořt et al. [2]. This region contains predominantly ascending flow along the vessel axis towards the impeller.

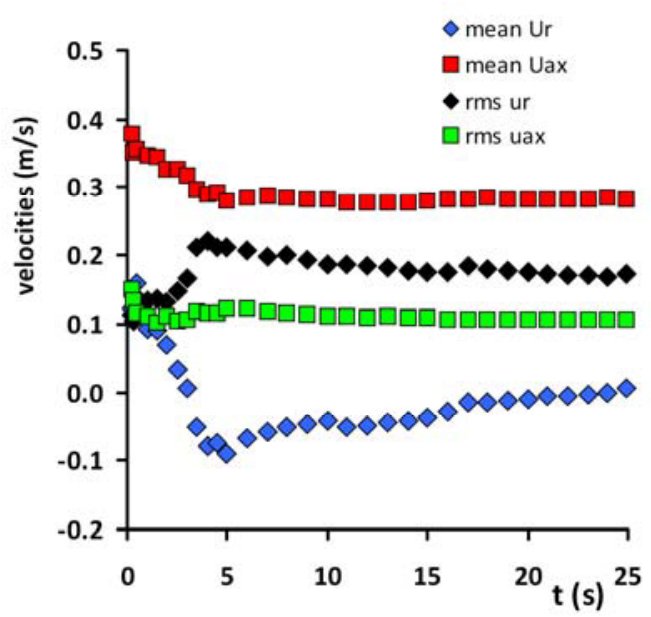

Fig. 2. Velocities vs. time length of averaging interval w-N300; Re = 95102 .

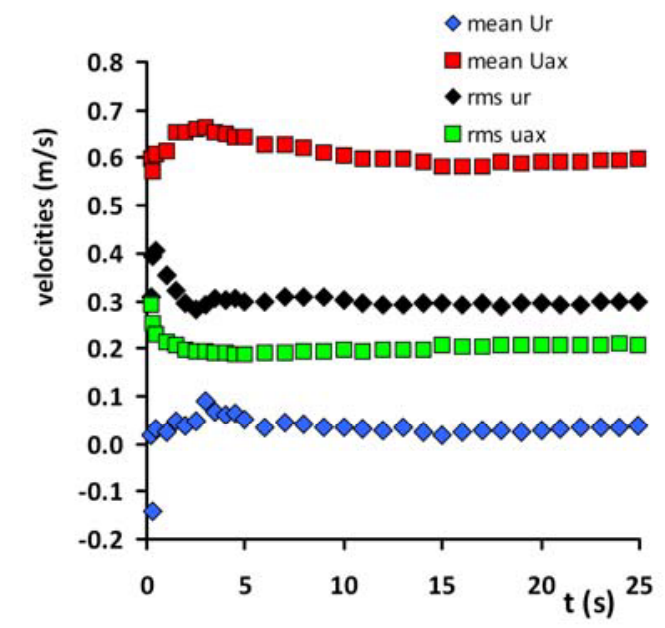

Fig. 3. Velocities vs. time length of averaging interval W-N600; Re = 189292 .

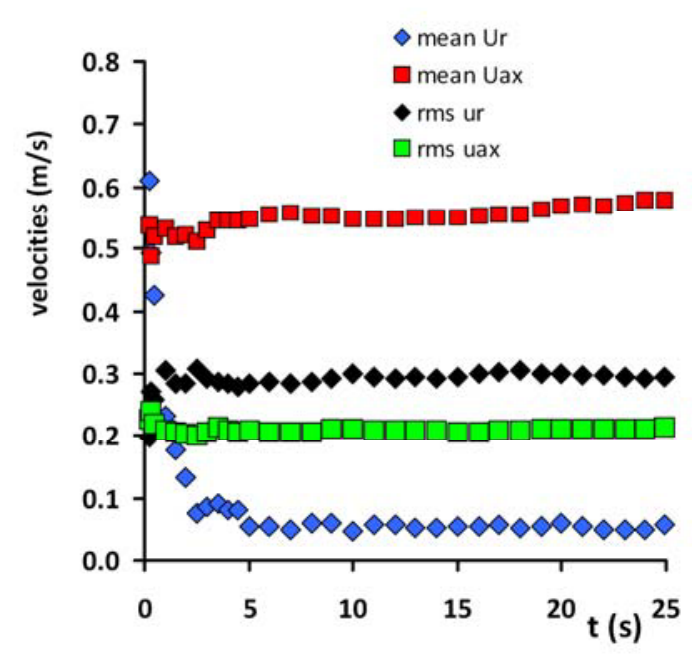

Fig. 4. Velocities vs. time length of averaging interval g28-N600; $\mathrm{Re}=88445$.

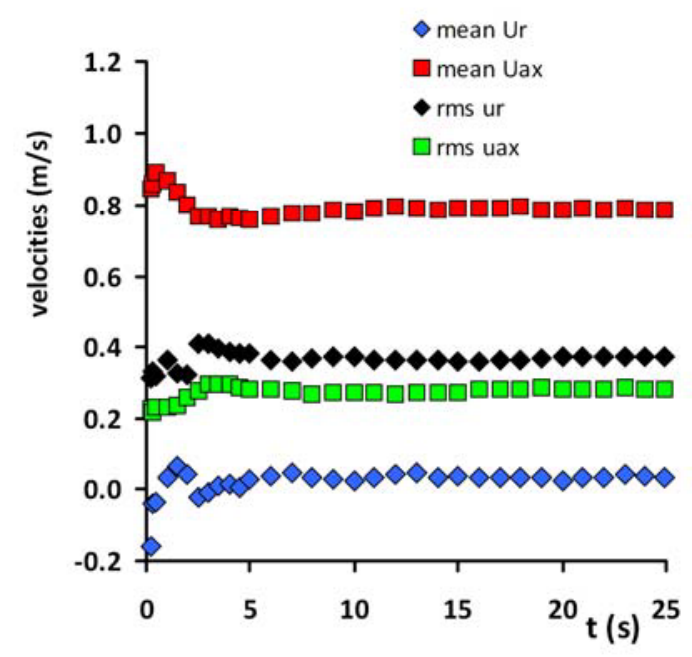

Fig. 5. Velocities vs. time length of averaging interval g28-N750; Re = 110556 . 


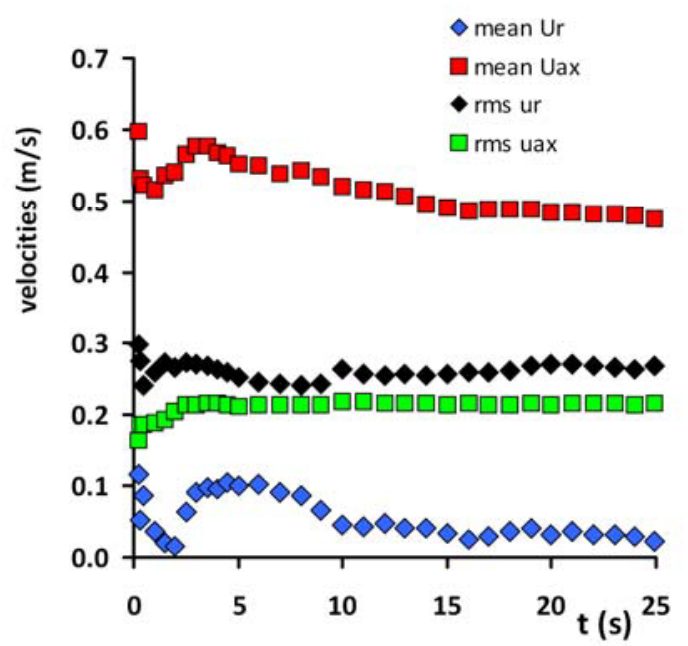

Fig. 6. Velocities vs. time length of averaging interval g43-N600; $\mathrm{Re}=58963$.

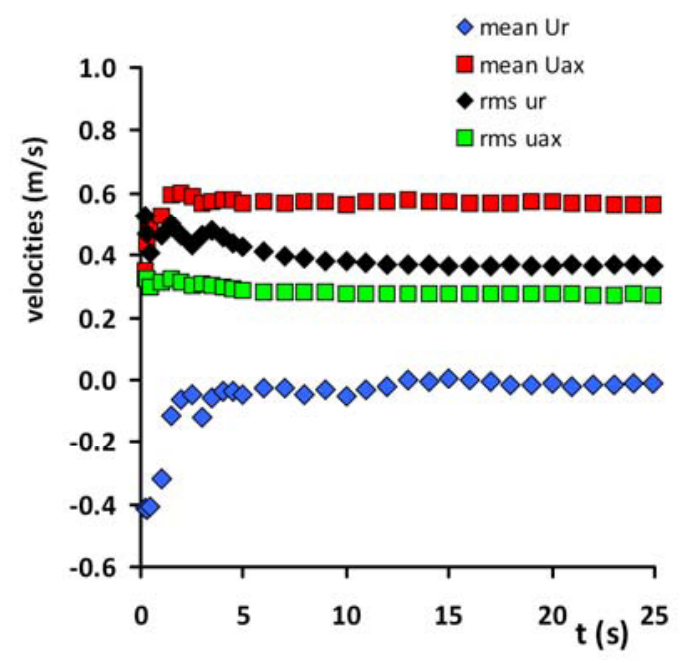

Fig. 7. Velocities vs. time length of averaging interval g43-N750; Re = 73704 .

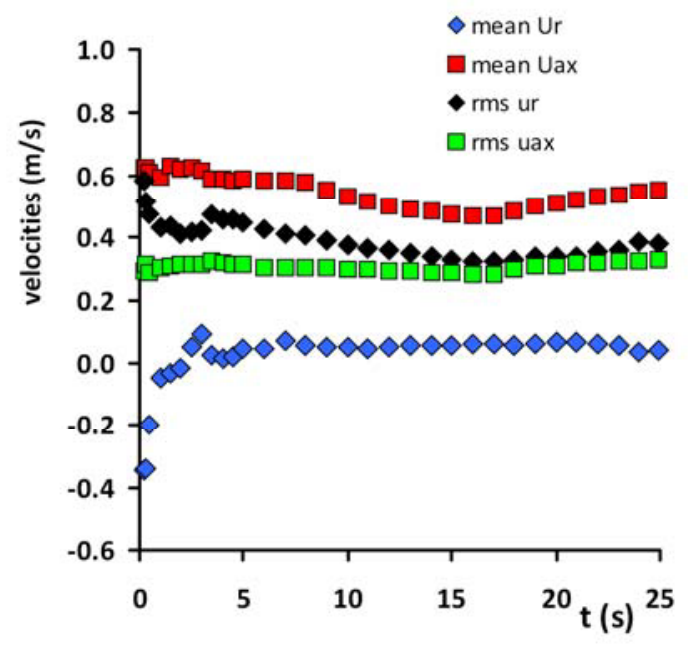

Fig. 8. Velocities vs. time length of averaging interval g43-N850; $\mathrm{Re}=85531$.

The time length of averaging interval that is necessary to stabilize the velocity line (i.e. to reach the stable velocity value) is called the stabilization time.
Thus principally, the minimum record time must the same or longer than this stabilization time.

For determination of stabilization time the same method that is used for determination of the blending time [3] was used. Using this method the stabilization time was found at the moment when the volatility of time dependence of time-averaged velocity is less than defined percentage of stable value. Usually, the $\pm 2 \%$ range is applied. The principle of evaluation of stabilization time is demonstrated in Fig. 9 for illustration.

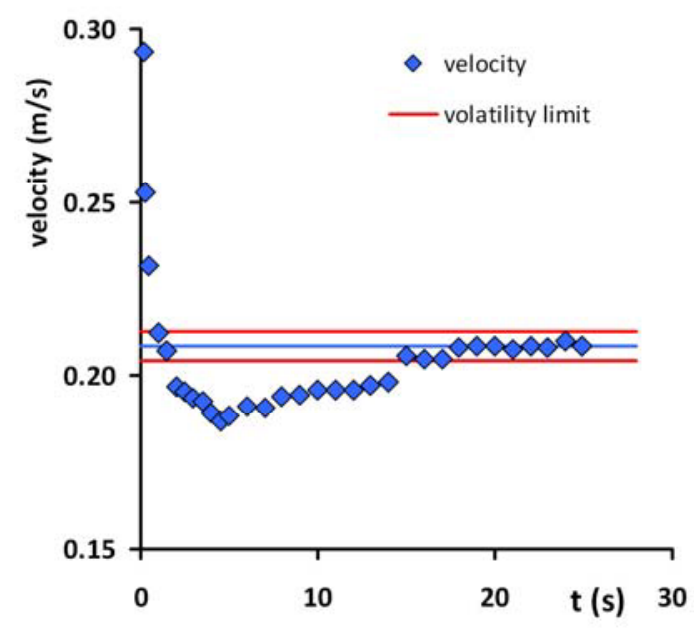

Fig. 9. Example of evaluation of stabilization time.

The stabilization time was determined for each velocity (i.e. for the mean- and fluctuation velocities in radial and axial direction) separately. In some cases (radial mean velocity for $\mathrm{w}-\mathrm{N} 300$ and g43-N600) the velocity time course was not stabilized within whole measured record time. In these cases the stabilization time was roughly estimated however is not taken into account during following treatment. In some other cases (e.g. axial mean velocity and fluctuation velocities for g43-N850) the time courses exhibit quasi-stable wave character instead of stable line. In these cases the stabilization time could not be determined.

The stabilization times determined are presented in Table 2 in dimensionless form as a product of time and impeller speed for each velocity and experimental run. The dimensionless time defined as a product of impeller rotational speed and time corresponds to total number of rounds of the impeller during given time.

The effect of time length of averaging interval on calculated time-averaged velocity can be spectacularly demonstrated plotting data in dimensionless form. The dependences of dimensionless velocity on dimensionless time length of averaging velocity are shown for illustration in Figures 10 and 11 for the radial mean velocity and axial fluctuation velocity, respectively.

As follows from Table 2 the values of dimensionless stabilization time oscillate in the range from 25 in minimum value to 188 in maximum value depending on impeller rotational speed and viscosity, i.e. depending on the impeller Reynolds number. 
Table 2. Dimensionless stabilization time N.t. $t_{S}$ for $\pm 2 \%$ volatility

\begin{tabular}{|c|c|c|c|c|c|}
\hline Run \# & $\begin{array}{c}\text { Re } \\
(-)\end{array}$ & $\bar{U}_{r}$ & $\bar{U}_{a x}$ & $\bar{u}_{r}$ & $\bar{u}_{a x}$ \\
\hline w-N300 & 94641 & 110 & 25 & 100 & 80 \\
w-N600 & 189282 & 100 & 100 & 110 & 150 \\
g28-N600 & 88445 & 50 & 100 & 100 & 50 \\
g28-N750 & 110556 & 100 & 100 & 125 & 188 \\
g43-N600 & 58963 & 150 & 160 & 100 & 30 \\
g43-N750 & 73704 & 163 & 38 & 150 & 100 \\
g43-N850 & 83531 & 142 & n.a. & n.a. & n.a. \\
\hline
\end{tabular}

Note: Values printed by italic letters were excluded from data treatment.

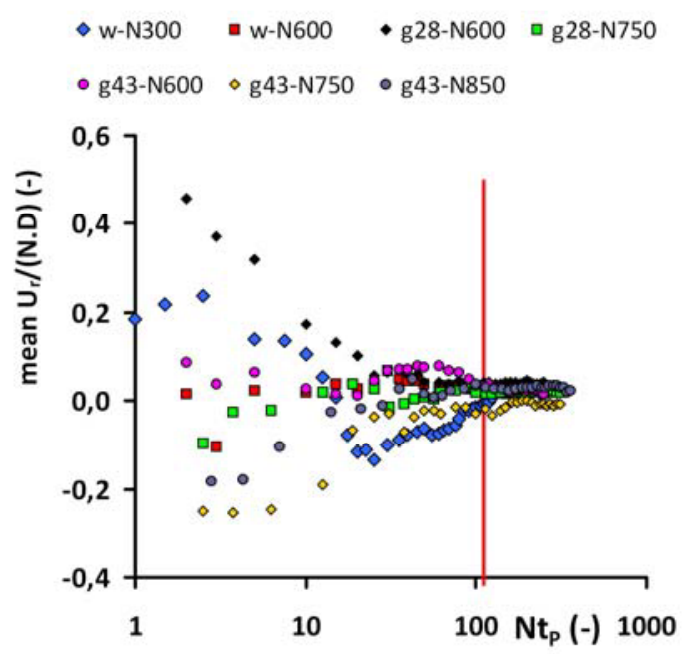

Fig. 10. Dimensionless velocity as a function of dimensionless time length of averaging interval - radial mean velocity

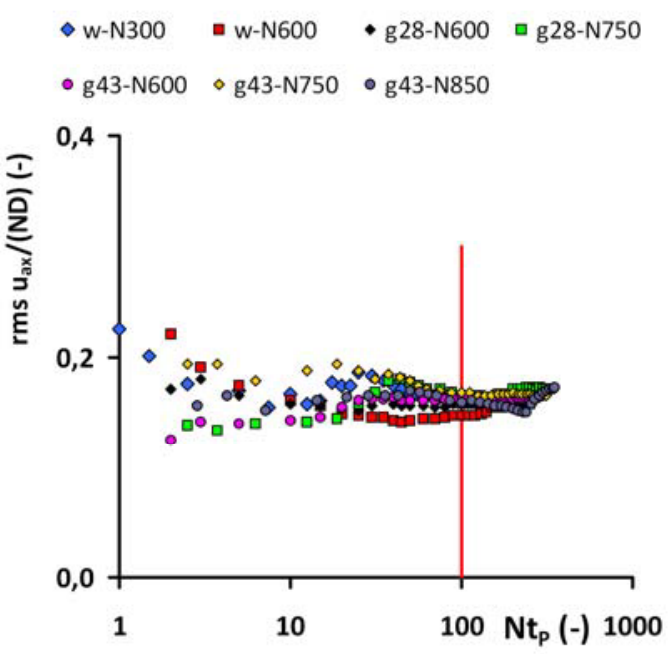

Fig. 11. Dimensionless velocity as a function of dimensionless time length of averaging interval - axial fluctuation velocity

Table 3. Dimensionless stabilization time for $\pm 2 \%$ volatility - effect of the impeller Reynolds number

\begin{tabular}{|c|c|c|c|c|c|}
\hline Velocity & $\begin{array}{c}\mathrm{m} \\
(-)\end{array}$ & $\begin{array}{c}\mathrm{t} \text {-distribution } \\
\mathrm{t}_{(\mathrm{m}-2), \alpha=0.05}\end{array}$ & $\begin{array}{c}\text { Relation } \\
\mathrm{N} . \mathrm{t}_{\mathrm{S}}=\mathrm{B}_{\mathrm{R}} \cdot(\mathrm{Re})^{\beta} \\
\beta_{\text {calc }}(-)\end{array}$ & $\begin{array}{c}\mathrm{t} \text {-characteristics }|\mathrm{t}| \\
\text { Hypothesis } \\
\left.\text { N.t } \mathrm{t}_{\mathrm{S}}=\mathrm{B}_{\mathrm{H} \cdot(\mathrm{Re})}\right)^{0}\end{array}$ & $\begin{array}{c}\text { Average } \\
\text { value }\end{array}$ \\
\hline $\bar{U}_{r}$ & 5 & 3.1825 & -0.231 & 0.3 (acceptable) & $111 \pm 54$ \\
\hline $\bar{U}_{a x}$ & 6 & 2.7764 & 0.047 & 0.1 (acceptable) & $87 \pm 52$ \\
\hline $\bar{u}_{r}$ & 6 & 2.7764 & -0.010 & 0.1 (acceptable) & $114 \pm 21$ \\
\hline $\bar{u}_{a x}$ & 6 & 2.7764 & 1.258 & 2.2 (acceptable) & $100 \pm 63$ \\
\hline Overall data & 23 & 2.0796 & 0.319 & 1.01 (acceptable) & $103 \pm 19$ \\
\hline
\end{tabular}

Note: The t-distribution for (m-2) degrees of freedom and significance level $\alpha=0.05$.

The effect of impeller Reynolds number on dimensionless stabilization time was tested by hypothesis testing ([4]) for each velocity separately. The statistical method of hypothesis testing can estimate whether the differences between the predicted parameter values (e.g. according to some proposed theory) and whether the parameter values evaluated from the measured data are negligible. In this case, we assumed dependence of the tested property on the impeller rotational speed, described by the simple power law correlation property $=B \cdot R e^{\beta}$, and then the difference between predicted exponent $\beta_{\text {pred }}$ and evaluated exponent $\beta_{\text {calc }}$ was tested. The hypothesis test characteristics are given as $t=\left(\beta_{\text {calc }}-\beta_{\text {pred }}\right) / s_{\beta}$ where $\mathrm{s}_{\beta}$ is the standard error of parameter $\beta_{\text {calc }}$. If the calculated $|t|$ value is less 
than the critical value of the t-distribution for $(m-2)$ degrees of freedom and significance level $\alpha$, the difference between $\beta_{\text {calc }}$ and $\beta_{\text {pred }}$ is statistically negligible (statisticians state: "the hypothesis cannot be rejected"). In our case, the independence of dimensionless stabilization time from the impeller Reynolds number was tested as the hypothesis, i.e. $N . t_{S}=$ $B .(R e)^{0}=$ const., i.e. $\beta_{\text {pred }}=0$. The hypothesis test results are presented in Table 3. For illustration, the values of calculated $|t|$ value are presented here also.

On the basis of the results of this hypothesis test, we assume that dimensionless stabilization times determined for each velocity separately can be statistically taken as constant and independent of the impeller Reynolds number. The average values were calculated and are presented in Table 3.

Since the values of dimensionless stabilization time $N . t_{S}$ are comparable regardless of the velocity type and velocity direction, we can assume that the stabilization time should be the same for both the mean- and fluctuation velocity in both radial and axial direction. Assuming the independency of dimensionless stabilization time on velocity type we evaluated all data together. The results are presented in Table 3.

The independency on impeller Reynolds number was confirmed and averaged value of $N . t_{S}=103 \pm 19$ was calculated. It means that the minimum record time must cover at least 103 rounds of the impeller to obtain relevant results of velocity field.

For comparison, by the same procedure the dimensionless stabilization time $N . t_{S}=81 \pm 16$ can be determined for $\pm 5 \%$

\section{Conclusions}

The following results have been obtained:

The hydrodynamics and flow field were investigated in a vessel $400 \mathrm{~mm}$ in the inner diameter agitated by a Rushton turbine using the 2-D Time Resolved Particle Image Velocimetry (2-D TR PIV). The velocity fields were measured in the zone in upward flow to the impeller for impeller rotation speeds from $300 \mathrm{rpm}$ to $850 \mathrm{rpm}$ and three liquids of different viscosities, corresponding to the impeller Reynolds number in the range $50000<\operatorname{Re}<189000$.

The effect of time length of the averaging interval on calculated time-averaged velocity was investigated. The time courses of mean- and fluctuation velocities in radial and axial direction calculated for averaging intervals of various time lengths were calculated in selected position in agitated system for each experimental run.

Further, the stabilization time (the time length of averaging interval that is necessary to stabilize the velocity line) was determined for the mean- and fluctuation velocities in radial and axial direction and for each experimental run. Principally, the minimum record time must be the same or longer than this stabilization time.

On the basis of the statistical analysis it was found that minimum record time $t_{R \min }$ must satisfied the following condition $N . t_{R \min } \propto N . t_{S}=103 \pm 19$ for the investigated area and $\pm 2 \%$ volatility.

This research has been supported by Grant Agency of the Czech Republic project No. 16-20175S and by Ministry of Education, Youth and Sports of the Czech Republic project No. LO1201 (National Programme for Sustainability I) and RVO:67985874.

\section{Symbols}

B baffle width, $\mathrm{m}$

C impeller clearance, $\mathrm{m}$

D impeller diameter, $\mathrm{m}$

$\mathrm{H}$ liquid height, $\mathrm{m}$

m number of experimental points, -

$\mathrm{N}$ impeller rotational speed, $1 / \mathrm{s}$

$\mathrm{N}_{\mathrm{P}}$ number of data items in velocity data set for averaging interval, -

$\mathrm{N}_{\mathrm{R}}$ number of data in velocity data set, -

$\mathrm{r}$ radius (radial coordinate), $\mathrm{m}$

$r^{*} \quad$ dimensionless radius; $r^{*}=2 r / T$, -

Re impeller Reynolds number; $\mathrm{Re}=\mathrm{N} . \mathrm{D}^{2} / v$, -

$\mathrm{t}$ time, $\mathrm{s}$

$t_{i} \quad$ observation time, $s$

$t_{P} \quad$ time length of averaging interval, $s$

$t_{R}$ record time, $s$

$t_{S} \quad$ stabilization time, $s$

$t_{(m-2), a}=0.05 \quad t$-distribution for $(m-2)$ degrees of freedom and significance level $\alpha$, -

$\mathrm{T}$ tank diameter, $\mathrm{m}$

$\mathrm{u}_{\mathrm{i}} \quad$ fluctuation velocity in $\mathrm{i}^{\text {th }}$ direction, $\mathrm{m} / \mathrm{s}$

$\bar{u}_{i} \quad$ root mean squared fluctuation velocity in $i^{\text {th }}$ direction, $\mathrm{m} / \mathrm{s}$

$\mathrm{U}_{\mathrm{i}} \quad$ instantaneous velocity in $\mathrm{i}^{\text {th }}$ direction, $\mathrm{m} / \mathrm{s}$

$\bar{U}_{i} \quad$ mean velocity in $\mathrm{i}^{\text {th }}$ direction, $\mathrm{m} / \mathrm{s}$

$\mathrm{z} \quad$ actual height (axial coordinate), $\mathrm{m}$

$\mathrm{z}^{*} \quad$ dimensionless height; $\mathrm{z}^{*}=\mathrm{z} / \mathrm{T}$, -

\section{References}

1. B. Kysela, J. Konfršt, Z. Chára, M. Kotek, EPJ Web of Conferences 67, 02065 (2014)

2. I. Fořt, A. Obeid, V. Březina, Coll. Czechoslov. Chem. Comm., 47, 226-239 (1982)

3. I. Fořt, T. Jirout, F. Rieger, R. Allner, R. Sperling, Acta Polytechnica, 41, 7-13 (2001)

4. B.L. Bowerman, R.T.O'Connell, Applied statistics: improving business processes (Richard D. Irwin, USA, 1997) 